PROCEEDINGS OF THE

AMERICAN MATHEMATICAL SOCIETY

Volume 125, Number 3, March 1997, Pages 785-793

S 0002-9939(97)03586-7

\title{
SHIFT-INVARIANT SPACES ON THE REAL LINE
}

\author{
RONG-QING JIA
}

(Communicated by J. Marshall Ash)

\begin{abstract}
We investigate the structure of shift-invariant spaces generated by a finite number of compactly supported functions in $L_{p}(\mathbb{R})(1 \leq p \leq \infty)$. Based on a study of linear independence of the shifts of the generators, we characterize such shift-invariant spaces in terms of the semi-convolutions of the generators with sequences on $\mathbb{Z}$. Moreover, we show that such a shiftinvariant space provides $L_{p}$-approximation order $k$ if and only if it contains all polynomials of degree less than $k$.
\end{abstract}

\section{INTRODUCTION}

The purpose of this paper is to investigate the structure of shift-invariant spaces on the real line. In particular, we are interested in those properties of shift-invariant spaces on the real line which are not shared by shift-invariant spaces on higher dimensional spaces $\mathbb{R}^{s}, s>1$.

Finitely generated shift-invariant subspaces of $L_{2}\left(\mathbb{R}^{s}\right)$ were studied in [4] by de Boor, DeVore, and Ron, who gave a simple characterization for such spaces in terms of the Fourier transforms of their generators. However, when $p \neq 2$, few results have been known for shift-invariant subspaces of $L_{p}\left(\mathbb{R}^{s}\right)$.

In this paper, we are mainly concerned with shift-invariant spaces generated by a finite number of compactly supported functions in $L_{p}(\mathbb{R})(1 \leq p \leq \infty)$. We will give a characterization for such spaces in terms of the semi-convolutions of their generators with sequences on $\mathbb{Z}$. The result is then applied to give a characterization of the approximation order provided by such shift-invariant spaces.

Let $S$ be a linear space of distributions on $\mathbb{R}$. We say that $S$ is shift-invariant if

$$
f \in S \Rightarrow f(\cdot-j) \in S \quad \forall j \in \mathbb{Z} .
$$

A mapping from $\mathbb{Z}$ to $\mathbb{C}$ is called a sequence. The linear space of all sequences on $\mathbb{Z}$ is denoted by $\ell(\mathbb{Z})$. Let $\phi$ be a compactly supported distribution on $\mathbb{R}$, and let $a: \mathbb{Z} \rightarrow \mathbb{C}$ be a sequence. The semi-convolution of $\phi$ with $a$, denoted $\phi *^{\prime} a$, is defined by

$$
\phi *^{\prime} \quad a:=\sum_{j \in \mathbb{Z}} \phi(\cdot-j) a(j) .
$$

Received by the editors April 13, 1995 and, in revised form, August 10, 1995.

1991 Mathematics Subject Classification. Primary 41A25, 41A15, 46E30.

Key words and phrases. Shift-invariant spaces, approximation order.

The author was supported in part by NSERC Canada under Grant OGP 121336.

(C)1997 American Mathematical Society 
Given a finite collection $\Phi$ of compactly supported distributions on $\mathbb{R}$, we denote by $S_{0}(\Phi)$ the linear span of $\{\phi(\cdot-j): \phi \in \Phi, j \in \mathbb{Z}\}$, and by $S(\Phi)$ the linear space of all distributions of the form $\sum_{\phi \in \Phi} \phi *^{\prime} a_{\phi}$ with $a_{\phi}$ being a sequence on $\mathbb{Z}$ for each $\phi \in \Phi$. The elements in $\Phi$ are called the generators for $S(\Phi)$.

Now suppose that $\Phi$ is a finite subset of $L_{p}(\mathbb{R})$ for some $p$ with $1 \leq p \leq \infty$. We denote by $S_{p}(\Phi)$ the closure of $S_{0}(\Phi)$ in $L_{p}(\mathbb{R})$. One of the main results of this paper is a characterization of $S_{p}(\Phi)$ in terms of semi-convolution. In Section 3, we shall prove that for $1<p<\infty$, a function $f \in L_{p}(\mathbb{R})$ lies in $S_{p}(\Phi)$ if and only if

$$
f=\sum_{\phi \in \Phi} \phi *^{\prime} a_{\phi}
$$

for some sequences $a_{\phi} \in \ell(\mathbb{Z})$. When $p=\infty$, a modified result will also be established.

We observe that this result is not valid for the case $p=1$. To see this, let $\chi$ be the characteristic function of the interval $[0,1)$, and let $\phi:=\chi-\chi(\cdot-1)$. Then for any $f \in S_{1}(\phi)$ we have $\int f=0$; hence $\chi \notin S_{1}(\phi)$. But $\chi=\sum_{j=0}^{\infty} \phi(\cdot-j)$.

Next, we consider approximation in $L_{p}(\mathbb{R})$ spaces $(1 \leq p \leq \infty)$. For $f, g \in L_{p}(\mathbb{R})$, we write $\operatorname{dist}_{p}(f, g)$ for $\|f-g\|_{p}$. Moreover, for a subset $G$ of $L_{p}(\mathbb{R})$, the distance from $f$ to $G$, denoted $\operatorname{dist}_{p}(f, G)$, is defined by

$$
\operatorname{dist}_{p}(f, G):=\inf _{g \in G}\|f-g\|_{p} .
$$

Let $\Phi$ be a finite collection of compactly supported functions in $L_{p}(\mathbb{R})$. The preceding result tells us that $S_{p}(\Phi)=S(\Phi) \cap L_{p}(\mathbb{R})$ for $1<p<\infty$. Suppose $1 \leq p \leq \infty$. Let $S:=S(\Phi) \cap L_{p}(\mathbb{R})$, and let $S^{h}:=\{g(\cdot / h): g \in S\}$ for $h>0$. Given a real number $r \geq 0$, we say that $S(\Phi)$ provides $L_{p^{-}}$approximation order $r$ if, for each sufficiently smooth function $f \in L_{p}(\mathbb{R})$,

$$
\operatorname{dist}_{p}\left(f, S^{h}\right) \leq C h^{r},
$$

where $C$ is a positive constant independent of $h(C$ may depend on $f$ ). We say that $S(\Phi)$ provides $L_{p^{-}}$density order $r$ (see [3]) if, for each sufficiently smooth function $f \in L_{p}(\mathbb{R})$,

$$
\lim _{h \rightarrow 0^{+}} \operatorname{dist}_{p}\left(f, S^{h}\right) / h^{r}=0 .
$$

In [7] Jia characterized the $L_{\infty}$-approximation order of $S(\Phi)$ in terms of the Strang-Fix conditions (see [16]). When $\Phi$ consists of a single generator $\phi$, Ron [13] proved that, for a positive integer $k, S(\phi)$ provides $L_{\infty}$-approximation order $k$ if and only if $S(\phi)$ contains $\Pi_{k-1}$, the set of all polynomials of degree $\leq k-1$. Zhao [18] also gave a characterization for the $L_{p}$-approximation order $(1<p<\infty)$ provided by $S(\phi)$.

In Section 4 , we shall prove that $S(\Phi)$ provides $L_{p}$-approximation order $(1 \leq$ $p \leq \infty)$ if and only if $S(\Phi)$ contains $\Pi_{k-1}$. This result is no longer true for shiftinvariance spaces on $\mathbb{R}^{s}, s>1$. See the counterexamples given in [5] and [6].

In our study of shift-invariant spaces linear independence plays a crucial role. Let $\Phi$ be a finite collection of compactly supported distributions on $\mathbb{R}$. The shifts of the elements in $\Phi$ are said to be linearly independent if

$$
\sum_{\phi \in \Phi} \phi *^{\prime} a_{\phi}=0 \Rightarrow a_{\phi}=0 \quad \forall \phi \in \Phi
$$


When the shifts of the elements in $\Phi$ are linearly independent, we say that $S(\Phi)$ has linearly independent generators.

In Section 2 we shall show that a finitely generated shift-invariant space always has linearly independent generators. More precisely, if $\Phi$ is a finite collection of compactly supported distributions on $\mathbb{R}$, then there exists a finite collection $\Psi$ of compactly supported distributions on $\mathbb{R}$ such that $S(\Psi)=S(\Phi)$ and the shifts of the elements in $\Psi$ are linearly independent. When $\Phi$ consists of compactly supported continuous functions, this result was essentially known to de Boor and DeVore (see [2]). When $\Phi$ consists of a single generator $\phi$, Ron [12] showed that $S(\phi)$ contains a linearly independent generator. Our contribution is to give a concrete construction for $\Psi$ so that $\Psi$ inherits most properties possessed by $\Phi$. For instance, if $\Phi \subset L_{p}(\mathbb{R})$ for some $p$ with $1 \leq p \leq \infty$, then $\Psi$ can be chosen to be a subset of $L_{p}(\mathbb{R})$. Furthermore, for $1<p \leq \infty, \Psi$ can be chosen to be a subset of $S_{p}(\Phi)$. These properties enable us to characterize shift-invariant subspaces of $L_{p}(\mathbb{R})$ and the approximation order provided by them.

\section{LINEAR INDEPENDENCE}

This section is devoted to a study of linear independence. Linear independence can be characterized in terms of the Fourier transforms of the generators. For a compactly supported integrable function $f$ on $\mathbb{R}$, the Fourier-Laplace transform of $f$ is given by

$$
\hat{f}(\xi):=\int_{\mathbb{R}} f(x) e^{-i x \xi} d x, \quad \xi \in \mathbb{C}
$$

The domain of the Fourier-Laplace transform can be extended to all compactly supported distributions. If $f$ is a compactly supported distribution, then $\hat{f}: \xi \mapsto$ $\hat{f}(\xi)$ is an entire function on $\mathbb{C}$. It is known (see [10] and the references cited there) that the shifts of the elements in $\Phi$ are linearly independent if and only if for every $\zeta \in \mathbb{C}$, the sequences $(\hat{\phi}(\zeta+2 \pi k))_{k \in \mathbb{Z}}, \phi \in \Phi$, are linearly independent.

For later use we introduce some concepts related to compactly supported distributions. Let $\phi$ be a compactly supported distribution on $\mathbb{R}$. Suppose $\phi \neq 0$. The support of $\phi$, denoted supp $\phi$, is a compact subset of $\mathbb{R}$. Let $\left[r_{\phi}, s_{\phi}\right]$ be the smallest integer-bounded interval containing supp $\phi$. The length of the interval $\left[r_{\phi}, s_{\phi}\right]$ is

$$
l(\phi):=s_{\phi}-r_{\phi}
$$

We call $l(\phi)$ the length of $\phi$.

Let $\Phi$ be a finite collection of compactly supported distributions on $\mathbb{R}$. The length of $\Phi$, denoted $l(\Phi)$, is defined by

$$
l(\Phi):=\sum_{\phi \in \Phi} l(\phi) .
$$

Also, we denote by $\# \Phi$ the number of elements in $\Phi$.

Theorem 1. Let $\Phi$ be a finite collection of nontrivial distributions on $\mathbb{R}$ with compact support. Then there exists a finite collection $\Psi$ of compactly supported distributions on $\mathbb{R}$ with the following properties:

(a) The shifts of the elements in $\Psi$ are linearly independent;

(b) $\# \Psi \leq \# \Phi$

(c) $\Phi \subset S_{0}(\Psi)$;

(d) $S(\Psi)=S(\Phi)$. 
If, in addition, $\Phi \subset L_{p}(\mathbb{R})$ for some $p, 1 \leq p \leq \infty$, then $\Psi$ can be chosen to be a subset of $L_{p}(\mathbb{R})$. Furthermore, for $1<p \leq \infty, \Psi$ can be chosen to be a subset of $S_{p}(\Phi)$.

Proof. It is sufficient to prove that, if the shifts of the elements in $\Phi$ are linearly dependent, then there exists $\Psi$ with $l(\Psi) \leq l(\Phi)-1$ satisfying all the conclusions of the theorem, except perhaps (a). Suppose $\Phi=\left\{\phi_{1}, \ldots, \phi_{m}\right\}$. Let

$$
K(\Phi):=\left\{\left(b_{1}, \ldots, b_{m}\right) \in(\ell(\mathbb{Z}))^{m}: \sum_{j=1}^{m} \phi_{j} *^{\prime} b_{j}=0\right\} .
$$

Then the shifts of the elements in $\Phi$ are linearly independent if and only if $K(\Phi)=$ $\{0\}$. If $K(\Phi)=\{0\}$, then we may take $\Psi=\Phi$. Suppose $K(\Phi) \neq\{0\}$. By [10, Theorem 3.3], $K(\Phi) \neq\{0\}$ implies that there exists some $\theta \in \mathbb{C} \backslash\{0\}$ and $\left(a_{1}, \ldots, a_{m}\right) \in \mathbb{C}^{m} \backslash\{0\}$ such that

$$
\left(a_{1} \theta^{()}, \ldots, a_{m} \theta^{()}\right) \in K(\Phi),
$$

where $\theta^{()}$denotes the sequence $k \mapsto \theta^{k}, k \in \mathbb{Z}$. It follows from (2.1) that

$$
\sum_{j=1}^{m} \sum_{k=-\infty}^{\infty} a_{j} \theta^{k} \phi_{j}(\cdot-k)=0 .
$$

For each $\phi_{j}$, let $r_{j}:=r_{\phi_{j}}$ and $s_{j}:=s_{\phi_{j}}$. After shifting the $\phi_{j}$ appropriately, we may assume that all $r_{j}=0$. Then $s_{j}=l\left(\phi_{j}\right)$, the length of $\phi_{j}$. Let

$$
l:=\max \left\{l\left(\phi_{j}\right): a_{j} \neq 0\right\} .
$$

For simplicity, we assume that $a_{1} \neq 0$ and $l\left(\phi_{1}\right)=l$. Let

$$
\rho:=\sum_{j=1}^{m} a_{j} \phi_{j}
$$

and

$$
\psi:=\sum_{k=0}^{\infty} \theta^{k} \rho(\cdot-k) .
$$

By our choice of $\rho$, we deduce from (2.2) that

$$
\sum_{k=-\infty}^{\infty} \theta^{k} \rho(\cdot-k)=0
$$

Let $\Psi:=\left\{\psi, \phi_{2}, \ldots, \phi_{m}\right\}$. We have

$$
\psi-\theta \psi(\cdot-1)=\sum_{k=0}^{\infty} \theta^{k} \rho(\cdot-k)-\sum_{k=0}^{\infty} \theta^{k+1} \rho(\cdot-k-1)=\rho=a_{1} \phi_{1}+\cdots+a_{m} \phi_{m} .
$$

Since $a_{1} \neq 0$, we obtain $\phi_{1} \in S_{0}\left(\psi, \phi_{2}, \ldots, \phi_{m}\right)$, and hence $\Phi \subset S_{0}(\Psi)$. It follows that $S(\Phi) \subseteq S(\Psi)$.

Evidently, $\psi \in S(\Phi)$. If $f=\psi *^{\prime} b$ for some sequence $b$ on $\mathbb{Z}$, then for any bounded open interval $E$ of $\mathbb{R}$, there exists an element $g \in S(\Phi)$ such that $g$ agrees with $f$ on $E$. Thus, by [8, Theorem 4], $f$ belongs to $S(\Phi)$. This shows $S(\Psi) \subseteq S(\Phi)$. Therefore $S(\Psi)=S(\Phi)$.

Let us show $l(\Psi)<l(\Phi)$. For this purpose we only have to prove supp $\psi \subseteq$ $[0, l-1]$. Clearly, supp $\psi \subseteq[0, \infty)$. Hence, it suffices to show that $\langle\psi, u\rangle=0$ for 
every $u \in C_{c}^{\infty}(\mathbb{R})$ with supp $u \subset(l-1, \infty)$. Let $u$ be such a test function. Note that for each $j, \phi_{j}(\cdot-k)$ is supported on $[k, l+k]$. Hence $\left\langle\phi_{j}(\cdot-k), u\right\rangle=0$ for $k \leq-1$. It follows that $\langle\rho(\cdot-k), u\rangle=0$ for $k \leq-1$. This in connection with (2.4) gives

$$
\langle\psi, u\rangle=\left\langle\sum_{k=0}^{\infty} \theta^{k} \rho(\cdot-k), u\right\rangle=\left\langle\sum_{k=-\infty}^{\infty} \theta^{k} \rho(\cdot-k), u\right\rangle=0 .
$$

Consequently, supp $\psi \subseteq[0, l-1]$.

Now suppose $\Phi \subset \bar{L}_{p}(\mathbb{R})$ for some $p, 1 \leq p \leq \infty$. Then $\rho \in L_{p}(\mathbb{R})$, and (2.3) tells us that for each integer $k, \psi$ is $p$ th power integrable on the interval $[k, k+1]$. But $\psi$ is compactly supported; hence $\psi \in L_{p}(\mathbb{R})$.

It remains to prove that $\psi \in S_{p}(\Phi)$ if $\Phi \subset L_{p}(\mathbb{R})$ for $1<p \leq \infty$. If $|\theta|<1$, then (2.3) implies $\psi \in S_{p}(\Phi)$. If $|\theta|>1$, then $\psi-\theta \psi(\cdot-1)=\rho$ implies

$$
\psi=\sum_{k=1}^{\infty}-\theta^{-k} \rho(\cdot+k) \in S_{p}(\Phi) .
$$

When $|\theta|=1$, we set

$$
f_{n}:=\sum_{k=0}^{n-1}(1-k / n) \theta^{k} \rho(\cdot-k),
$$

where $n$ is an integer greater than $l$. Then $f_{n} \in S_{0}(\Phi)$. The desired result $\psi \in S_{p}(\Phi)$ will be established if we can show

$$
\left\|f_{n}-\psi\right\|_{p} \rightarrow 0 \quad \text { as } n \rightarrow \infty .
$$

To prove (2.5) we observe that $\rho$ is supported on $[0, l], \psi$ is supported on $[0, l-1]$, and $f_{n}$ is supported on $[0, n+l-1]$. For $x \in[0, l-1]$ we have

$$
\psi(x)-f_{n}(x)=\sum_{k=0}^{l-1}(k / n) \theta^{k} \rho(x-k) .
$$

Hence

$$
\left\|\psi-f_{n}\right\|_{L_{p}([0, l-1])} \rightarrow 0 \quad \text { as } n \rightarrow \infty .
$$

For $x \in[n-1, n+l-1]$, we have $\psi(x)=0$ and

$$
\psi(x)-f_{n}(x)=\sum_{k=n-l}^{n-1}-(1-k / n) \theta^{k} \rho(x-k) .
$$

But $|1-k / n| \leq l / n$ for $n-l \leq k \leq n-1$; hence

$$
\left\|\psi-f_{n}\right\|_{L_{p}([n-1, n+l-1])} \rightarrow 0 \quad \text { as } n \rightarrow \infty .
$$

It remains to prove

$$
\left\|\psi-f_{n}\right\|_{L_{p}([l-1, n-1])} \rightarrow 0 \quad \text { as } n \rightarrow \infty .
$$

For this purpose let $j$ be an integer in $[l-1, n-2]$. We observe that for almost every $x \in[j, j+1], \rho(x-k)=0$ for $k \notin(j-l, j+1)$, and hence by (2.4) we have

$$
\sum_{k=j-l+1}^{j} \theta^{k} \rho(x-k)=\sum_{k=-\infty}^{\infty} \theta^{k} \rho(x-k)=0 .
$$


Therefore, for almost every $x \in[j, j+1]$, we have

$$
\begin{aligned}
\psi(x)-f_{n}(x) & =(1-j / n) \sum_{k=j-l+1}^{j} \theta^{k} \rho(x-k)-\sum_{k=j-l+1}^{j}(1-k / n) \theta^{k} \rho(x-k) \\
& =\sum_{k=j-l+1}^{j} \frac{k-j}{n} \theta^{k} \rho(x-k) .
\end{aligned}
$$

But $|k-j| \leq l$ for $j-l+1 \leq k \leq j$. Consequently, (2.8) holds true for $p=\infty$. If $1<p<\infty$, then there exists a positive constant $C$ independent of $n$ such that

$$
\int_{[j, j+1]}\left|\psi(x)-f_{n}(x)\right|^{p} d x \leq C^{p} / n^{p}, \quad l-1 \leq j \leq n-2 .
$$

It follows that

$$
\int_{[l-1, n-1]}\left|\psi(x)-f_{n}(x)\right|^{p} d x \leq n C^{p} / n^{p}=C^{p} / n^{p-1} .
$$

This verifies (2.8) for $1<p<\infty$. Finally, (2.6), (2.7), and (2.8) together imply (2.5). We conclude that $\psi \in S_{p}(\Phi)$ for $1<p \leq \infty$.

The results obtained so far can be summarized as follows: If the shifts of the elements in $\Phi$ are linearly dependent, then we can find a collection $\Psi$ of distributions such that $\# \Psi \leq \# \Phi, l(\Psi)<l(\Phi), \Phi \subset S_{0}(\Psi)$, and $S(\Psi)=S(\Phi)$. Furthermore, if $\Phi \subset L_{p}(\mathbb{R})(1 \leq p \leq \infty)$, then $\Psi$ possesses the additional properties stated in the theorem. Repeat the preceding process until $l(\Psi)$ achieves its minimum. The resulting set $\Psi$ has the property that the shifts of the elements in $\Psi$ are linearly independent. Moreover, $\Psi$ meets the requirement of the theorem.

\section{Characterization OF Shift-InVARIANT SPACES}

In this section we investigate the structure of shift-invariant spaces generated by a finite number of compactly supported functions in $L_{p}(\mathbb{R})(1 \leq p \leq \infty)$.

We use $\ell_{0}(\mathbb{Z})$ to denote the linear space of all finitely supported sequences on $\mathbb{Z}$. Then, for $1 \leq p<\infty, \ell_{0}(\mathbb{Z})$ is dense in $\ell_{p}(\mathbb{Z})$. For $p=\infty$, the closure of $\ell_{0}(\mathbb{Z})$ in $\ell_{\infty}(\mathbb{Z})$ is $c_{0}(\mathbb{Z})$, the linear space of all sequences $a$ on $\mathbb{Z}$ such that $\lim _{|k| \rightarrow \infty} a(k)=0$. For a measurable subset $E$ of $\mathbb{R}$ and a measurable function $f$ on $\mathbb{R}$, we denote by $\|f\|_{\infty}(E)$ the essential supremum of $f$ on $E$. Let $L_{\infty, 0}(\mathbb{R})$ be the linear space of all functions $f \in L_{\infty}(\mathbb{R})$ for which $\lim _{r \rightarrow \infty}\|f\|_{\infty}(\mathbb{R} \backslash[-r, r])=0$.

Let $\Phi=\left\{\phi_{1}, \ldots, \phi_{m}\right\}$ be a finite collection of compactly supported functions in $L_{p}(\mathbb{R})$. We say that the shifts of the functions of $\Phi$ are stable, if there exist two positive constants $C_{1}$ and $C_{2}$ such that for any choice of sequences $a_{1}, \ldots, a_{m} \in$ $\ell_{p}(\mathbb{Z})$

$$
C_{1} \sum_{j=1}^{m}\left\|a_{j}\right\|_{\ell_{p}(\mathbb{Z})} \leq\left\|\sum_{j=1}^{m} \phi_{j} *^{\prime} a_{j}\right\|_{L_{p}(\mathbb{R})} \leq C_{2} \sum_{j=1}^{m}\left\|a_{j}\right\|_{\ell_{p}(\mathbb{Z})} .
$$

It was proved by Jia and Micchelli in [10] and [11] that the shifts of the functions in $\Phi$ are stable if and only if for every $\xi \in \mathbb{R}$, the sequences $\left(\hat{\phi}_{j}(\xi+2 \pi k)\right)_{k \in \mathbb{Z}}$, $j=1, \ldots, m$, are linearly independent. Thus, if the shifts of the functions in $\Phi$ are linearly independent, then they are stable. 
Consider the linear mapping $T_{\Phi} \operatorname{from}\left(\ell_{p}(\mathbb{Z})\right)^{m}$ to $L_{p}(\mathbb{R})$ given by

$$
T_{\Phi}\left(a_{1}, \ldots, a_{m}\right)=\sum_{j=1}^{m} \phi_{j} *^{\prime} a_{j}, \quad a_{1}, \ldots, a_{m} \in \ell_{p}(\mathbb{Z}) .
$$

If the shifts of the functions in $\Phi$ are stable, then $T_{\Phi}$ is a continuous mapping and the range of $T_{\Phi}$ is closed (see [14, p. 70]). Therefore, for $1 \leq p<\infty, S_{p}(\Phi)$ is the range of $T_{\Phi}$. In other words, for $1 \leq p<\infty, f$ lies in $S_{p}(\Phi)$ if and only if $f=\sum_{\phi \in \Phi} \phi *^{\prime} a_{\phi}$ for some sequences $a_{\phi} \in \ell_{p}(\mathbb{Z}), \phi \in \Phi$. In the case $p=\infty$, $f \in S_{\infty}(\Phi)$ if and only if $f=\sum_{\phi \in \Phi} \phi *^{\prime} a_{\phi}$ for some sequences $a_{\phi} \in c_{0}(\mathbb{Z}), \phi \in \Phi$.

In general, we have the following characterization for $S_{p}(\Phi)(1<p \leq \infty)$, where the stability condition is not assumed.

Theorem 2. Let $\Phi$ be a finite collection of compactly supported functions in $L_{p}(\mathbb{R})$. Then for $1 \leq p \leq \infty, S(\Phi) \cap L_{p}(\mathbb{R})$ is closed in $L_{p}(\mathbb{R})$. Moreover, for $1<p<\infty$,

$$
S(\Phi) \cap L_{p}(\mathbb{R})=S_{p}(\Phi) .
$$

In other words, for $1<p<\infty$, a function $f$ lies in $S_{p}(\Phi)$ if and only if $f \in L_{p}(\mathbb{R})$ and

$$
f=\sum_{\phi \in \Phi} \phi *^{\prime} a_{\phi}
$$

for some sequences $a_{\phi} \in \ell(\mathbb{Z})$. In the case $p=\infty, f \in S_{\infty}(\Phi)$ if and only if $f \in L_{\infty, 0}(\mathbb{R})$ and $(3.2)$ holds true for some sequences $a_{\phi} \in \ell(\mathbb{Z})$.

Proof. By Theorem 1, there exists a finite collection $\Psi \subset L_{p}(\mathbb{R})$ such that $S(\Psi)=$ $S(\Phi)$ and the shifts of the functions in $\Psi$ are linearly independent. Moreover, for $1<p \leq \infty, \Psi$ can be so chosen that $S_{p}(\Psi)=S_{p}(\Phi)$.

We first show that $S(\Phi) \cap L_{p}(\mathbb{R})$ is closed in $L_{p}(\mathbb{R})(1 \leq p \leq \infty)$. This can be derived from $[8$, Theorem 4]. Here we establish this result by using the dual functionals discussed in [1] and [17]. Suppose $\Psi=\left\{\psi_{1}, \ldots, \psi_{m}\right\}$. Let $f \in S(\Psi) \cap$ $L_{p}(\mathbb{R})$. Then

$$
f=\sum_{j=1}^{m} \psi_{j} *^{\prime} a_{j},
$$

where $a_{j} \in \ell(\mathbb{Z}), j=1, \ldots, m$. From [1] and [17] we see that there are functions $u_{1}, \ldots, u_{m} \in C_{c}^{\infty}(\mathbb{R})$ such that for $j, k=1, \ldots, m$ and $\alpha \in \mathbb{Z}$,

$$
\left\langle\psi_{j}, u_{k}(\cdot-\alpha)\right\rangle=\delta_{j k} \delta_{\alpha 0},
$$

where $\delta_{j k}$ stands for the Kronecker sign: $\delta_{j k}=1$ for $j=k$ and $\delta_{j k}=0$ for $j \neq k$. It follows that

$$
a_{j}(\alpha)=\left\langle f, u_{j}(\cdot-\alpha)\right\rangle, \quad \alpha \in \mathbb{Z} .
$$

Since $f \in L_{p}(\mathbb{R})$, we obtain $a_{j} \in \ell_{p}(\mathbb{Z})$ for $j=1, \ldots, m$ (see [11, Theorem 3.1]). Thus, by the discussion at the beginning of this section, $S(\Psi) \cap L_{p}(\mathbb{R})$ is closed in $L_{p}(\mathbb{R})$. But $S(\Phi)=S(\Psi)$. Hence $S(\Phi) \cap L_{p}(\mathbb{R})$ is closed in $\ell_{p}(\mathbb{R})$.

Furthermore, for $1 \leq p<\infty, S(\Psi) \cap L_{p}(\mathbb{R})=S_{p}(\Psi)$. But, for $1<p \leq \infty$, we have $S_{p}(\Psi)=S_{p}(\Phi)$. Therefore, (3.1) is true for $1<p<\infty$.

Finally, it is easily seen that $S_{\infty}(\Psi) \subseteq S(\Psi) \cap L_{\infty, 0}(\mathbb{R})$. If $f \in S(\Psi) \cap L_{\infty, 0}(\mathbb{R})$ has the expression as in (3.3), then it follows from (3.4) that $a_{j} \in c_{0}(\mathbb{Z})$ for $j=1, \ldots, m$. Hence $f \in S_{\infty}(\Psi)$. This shows that $S_{\infty}(\Psi)=S(\Psi) \cap L_{\infty, 0}(\mathbb{R})$. But $S(\Phi)=S(\Psi)$ 
and $S_{\infty}(\Phi)=S_{\infty}(\Psi)$. We therefore conclude that $S_{\infty}(\Phi)=S(\Phi) \cap L_{\infty, 0}(\mathbb{R})$. This verifies the last statement of the theorem.

\section{Approximation order}

We are now in a position to consider approximation in $L_{p}(\mathbb{R})$ spaces $(1 \leq p \leq \infty)$.

Theorem 3. Let $\Phi$ be a finite collection of compactly supported functions in $L_{p}(\mathbb{R})$, $1 \leq p \leq \infty$. Let $k$ be a positive integer. Then the following statements are equivalent.

(a) $S(\Phi)$ provides $L_{p}$-approximation order $k$.

(b) $S(\Phi)$ provides $L_{p}$-density order $k-1$.

(c) $S(\Phi)$ contains $\Pi_{k-1}$, the set of all polynomials of degree $\leq k-1$.

(d) $S(\Phi)$ contains a compactly supported function $\psi$ such that

$$
\sum_{\beta \in \mathbb{Z}} q(\beta) \psi(\cdot-\beta)=q \quad \forall q \in \Pi_{k-1} .
$$

Proof. It is obvious that (a) implies (b). It was proved in [8] that (b) implies (c). The implication (d) $\Rightarrow$ (a) is well known. See [9] for an explicit $L_{p}$-approximation scheme. It remains to prove (c) $\Rightarrow(d)$. By Theorem 1 , we may assume that the shifts of the functions in $\Phi$ are linearly independent. Suppose $\Phi=\left\{\phi_{1}, \ldots, \phi_{m}\right\}$.

Since the shifts of the functions in $\Phi$ are linearly independent, there exist test functions $u_{1}, \ldots, u_{m} \in C_{c}^{\infty}(\mathbb{R})$ such that

$$
\left\langle\phi_{r}(\cdot-\alpha), u_{s}(\cdot-\beta)\right\rangle=\delta_{r s} \delta_{\alpha \beta}, \quad r, s \in\{1, \ldots, m\}, \alpha, \beta \in \mathbb{Z} .
$$

By condition (c), $q \in S(\Phi)$ for $q \in \Pi_{k-1}$. Hence by (4.2) we have

$$
q=\sum_{j=1}^{m} \sum_{\alpha \in \mathbb{Z}} \phi_{j}(\cdot-\alpha)\left\langle q(\cdot+\alpha), u_{j}\right\rangle
$$

Let $\left(\ell_{r}: r=1, \ldots, k\right)$ be the Lagrange polynomials of degree $k-1$ for the points $1, \ldots, k$. Then, for any $q \in \Pi_{k-1}$,

$$
q=\sum_{\alpha \in \mathbb{Z}} \sum_{j=1}^{m} \phi_{j}(\cdot-\alpha)\left\langle\sum_{r=1}^{k} q(r+\alpha) \ell_{r}, u_{j}\right\rangle=\sum_{\beta \in \mathbb{Z}} \psi(\cdot-\beta) q(\beta)
$$

with

$$
\psi:=\sum_{j=1}^{m} \sum_{r=1}^{k} \phi_{j}(r+\cdot)\left\langle\ell_{r}, u_{j}\right\rangle
$$

certainly a compactly supported element of $S(\Phi)$. Therefore, (c) implies (d).

It was proved by Schoenberg [15] that (4.1) is equivalent to the following conditions: $D^{\alpha} \hat{\psi}(0)=\delta_{\alpha 0}$ and $D^{\alpha} \hat{\psi}(2 \pi j)=0$ for $0 \leq \alpha<k$ and $j \in \mathbb{Z} \backslash\{0\}$. Now these conditions are referred to as the Strang-Fix conditions (see [16]). 


\section{REFERENCES}

1. A. Ben-Artzi and A. Ron, On the integer translates of a compactly supported function: dual bases and linear projectors, SIAM J. Math. Anal. 21 (1990), 1550-1562. MR 91j:41009

2. C. de Boor and R. DeVore, Partitions of unity and approximation, Proc. Amer. Math. Soc. 93 (1985), 705-709. MR 86f: 41003

3. C. de Boor, R. DeVore, and A. Ron, Approximation from shift-invariant subspaces of $L_{2}\left(\mathbb{R}^{d}\right)$, Trans. Amer. Math. Soc. 341 (1994), 787-806. MR 94d:41028

4. $\quad$, The structure of finitely generated shift-invariant subspaces in $L_{2}\left(\mathbb{R}^{d}\right)$, J. Functional Analysis 119 (1994), 37-78. MR 95g:46050

5. C. de Boor and K. Höllig, Approximation order from bivariate $C^{1}$-cubics: a counterexample, Proc. Amer. Math. Soc. 87 (1983), 649-655. MR 84j:41014

6. C. de Boor and R. Q. Jia, A sharp upper bound on the approximation order of smooth bivariate pp functions, J. Approx. Theory 72 (1993), 24-33. MR 94e:41012

7. R. Q. Jia, A characterization of the approximation order of translation invariant spaces, Proc. Amer. Math. Soc. 111 (1991), 61-70. MR 91d:41018

8. Trans. Amer. Math. Soc. 347 (1995), 2585-2594. CMP 94:13

9. R. Q. Jia and J. J. Lei, Approximation by multiinteger translates of functions having global support, J. Approx. Theory 72 (1993), 2-23. MR 94f:41024

10. R. Q. Jia and C. A. Micchelli, On linear independence of integer translates of a finite number of functions, Research Report CS-90-10 (1990), University of Waterloo. A revised version appeared in Proc. Edinburgh Math. Soc. 36 (1992), 69-85. MR 94e:41044

11. U Using the refinement equation for the construction of pre-wavelets II: Powers of two, Curves and Surfaces (P. J. Laurent, A. Le Méhauté, and L. L. Schumaker, eds.), Academic Press, New York, 1991, pp. 209-246. MR 92c:65006

12. A. Ron, Factorization theorem for univariate splines on regular grids, Israel J. Math. 70 (1990), 48-68. MR 91g:65025

13. $\ldots$ A characterization of the approximation order of multivariate spline spaces, Studia Math. 98 (1991), 73-90. MR 92g:41017

14. M. Schechter, Principles of Functional Analysis, Academic Press, New York, 1971. MR 56:3607

15. I. J. Schoenberg, Contributions to the problem of approximation of equidistant data by analytic functions, Quart. Appl. Math. 4 (1946), 45-99, 112-141. MR 8:55d

16. G. Strang and G. Fix, A Fourier analysis of the finite-element variational method, Constructive Aspects of Functional Analysis (G. Geymonat, ed.), C.I.M.E. (1973), 793-840.

17. K. Zhao, Global linear independence and finitely supported dual basis, SIAM J. Math. Anal. 23 (1992), 1352-1355. MR 93h:41034

18. _ Approximation from locally finite-dimensional shift-invariant spaces, Proc. Amer. Math. Soc. 124 (1996), 1857-1867. MR 96h:41019

Department of Mathematics, University of Alberta, Edmonton, Canada T6G 2G1

E-mail address: jia@xihu.math.ualberta.ca 